bioRxiv preprint doi: https://doi.org/10.1101/2020.12.15.422868; this version posted December 16, 2020. The copyright holder for this preprint

(which was not certified by peer review) is the author/funder. This article is a US Government work. It is not subject to copyright under 17 USC 105 and is also made available for use under a CCO license.

Running Head: Resting-state functional connectivity in XXY

\title{
Resting-state functional connectivity and psychopathology in Klinefelter syndrome (47, XXY)
}

Ethan T. Whitman ${ }^{1}$, Siyuan Liu ${ }^{1}$, Erin Torres ${ }^{1}$, Allysa Warling ${ }^{1}$, Kathleen Wilson ${ }^{1}$, Ajay Nadig ${ }^{1}$, Cassidy McDermott ${ }^{1}$, Liv S. Clasen ${ }^{1}$, Jonathan D. Blumenthal ${ }^{1}$, François M. Lalonde ${ }^{1}$, Stephen J. Gotts $^{2}$, Alex Martin ${ }^{2}$, and Armin Raznahan ${ }^{1}$

${ }^{1}$ Section on Developmental Neurogenomics, Human Genetics Branch, National Institute of Mental Health

${ }^{2}$ Section on Cognitive Neuropsychology, Laboratory of Brain and Cognition, National Institute of Mental Health

Corresponding Author: Armin Raznahan, razhanaha@mail.nih.gov

Tel: 301435792

Fax: 3014020296

Mailing address:

Building 10, Room 4N242

10 Center Drive

Bethesda, MD 20814 


\section{ABSTRACT}

Klinefelter syndrome (47, XXY; Henceforth: XXY syndrome) is a high impact but poorly understood genetic risk factor for neuropsychiatric impairment. Here, we provide the first neuroimaging study to map resting-state functional connectivity (rsFC) changes in XXY syndrome and ask how these might relate to brain anatomy and psychopathology. We collected resting state functional magnetic resonance imaging data from 75 individuals with XXY and 84 healthy XY males. We implemented a brain-wide screen to identify regions with altered global rsFC in XXY vs. XY males, and then used seed-based analysis to decompose these alterations. We further compared rsFC changes with regional changes in brain volume from voxel-based morphometry and tested for correlations between rsFC and symptom variation within XXY syndrome. We found that XXY syndrome was characterized by increased global rsFC in the left dorsolateral prefrontal cortex (DLPFC), associated with overconnectivity with diverse rsFC networks. Regional rsFC changes were partly coupled to regional volumetric changes in XXY syndrome. Within the precuneus, variation in DLPFC rsFC within XXY syndrome was correlated with the severity of psychopathology in XXY individuals. Our findings provide the first view of altered functional brain connectivity in XXY syndrome and delineate links between these alterations and those relating to both brain anatomy and psychopathology. Taken together, these insights advance biological understanding of XXY syndrome as a disorder in its own right, and as a model of genetic risk for psychopathology more broadly. 


\section{INTRODUCTION}

Klinefelter syndrome (47, XXY karyotype) is a sex chromosome aneuploidy disorder estimated to occur in approximately 1 in 633 male births [1]. This aneuploidy is associated with increased rates of neuropsychiatric disorders including autism, attention-deficit/hyperactivity disorder, and mood disorders relative to karyotypically normal males [2-5]. XXY syndrome increases risk for language difficulties alongside a modest decrease in general cognitive ability [2]. The biological bases of neuropsychiatric risk in XXY syndrome remain poorly understood, but there is extensive evidence for reproducible changes in regional brain anatomy from in vivo structural neuroimaging data. These changes include decreased total brain volume with relative contractions in temporal, frontal, and cerebellar structures and relative expansion in parietaloccipital regions [6-8]. The presence of overlapping regional anatomy changes in other disorders of X-chromosome dosage (e.g. trisomy X [9, 10]; and Turner syndrome $[9,10])$ suggests a direct role for X-linked genes in driving the observed anatomical brain changes in XXY syndrome.

Although there is a consensus regarding the spatial pattern of regional neuroanatomical changes in XXY syndrome, functional brain organization in XXY remains largely unstudied [11]. In contrast to several genetically defined neuropsychiatric disorders that are less common than XXY syndrome [e.g. 22q11.2 deletions [12-15], 16p11.2 copy number variations [13, 16], Fragile X disorder [17]], we still lack a brain-wide study of resting-state functional connectivity (rsFC) in XXY syndrome. Our study seeks to address this gap in knowledge with two main considerations in mind.

First, from the perspective of psychiatric genetics, XXY syndrome offers a powerful model for "genetics-first" research [18] into mechanisms of biological risk for psychopathology. Our study empirically considers three general questions regarding mechanisms of genetic risk in the 
human brain that remain largely unaddressed to date: (i) the degree to which genetically driven disruptions of brain structure and function are colocalized or differentially distributed across the brain [19], (ii) the degree to which the considerable variation in neuropsychiatric impairments within a neurogenetic disorder can be related to inter-individual variation in $\mathrm{rsFC}[20,21]$, and (iii) whether any such rsFC-behavior relationships amongst patients are colocalized with regions of altered $\mathrm{rFC}$ between patients and controls.

Second, the fact that XXY syndrome models X-chromosome dosage effects in humans means that better understanding brain changes in XXY syndrome would help address broader questions relating to X-chromosome biology. Specifically, because X-chromosome dosage disparity is a foundational genetic difference between males and females, $\mathrm{X}$-chromosome dosage effects are candidate contributors to sex differences in brain structure and function [22-27]. Furthermore, modelling X-chromosome dosage effects on rsFC through the study of XXY syndrome may help interpreting past observations that X-linked genes show elevated levels of brain expression relative to Y-linked and autosomal genes $[28,29]$ and an enrichment for genetic variants associated with intellectual disability syndromes [28, 29].

Motivated by these factors, we used resting-state functional magnetic resonance imaging (fMRI) to study rsFC in 75 males with XXY and 84 age-matched XY male controls. Our analytic approach first provides a brain-wide screen for regions with altered global rsFC in XXY, and then characterizes the patterns of interregional dysconnectivity that drive these effects [30]. We also test whether the spatial distribution of rsFC changes is related to that of regional brain volume changes. Finally, we examine whether the marked variability in neuropsychiatric outcome severity within $\mathrm{XXY}$ syndrome is related to variability in $\mathrm{rsFC}$, and whether any such relationships align with rsFC changes in XXY syndrome as compared to typically developing XY controls. 


\section{MATERIALS AND METHODS}

\section{Participants}

Participants were recruited to participate in this study through XXY parent support organizations, the National Institute of Mental Health (NIMH) website, and the National Institutes of Health (NIH) healthy volunteer office. The sample comprised 75 XXY participants (ages 6-25, mean age 16.7 years) and $84 \mathrm{XY}$ controls (ages 7-25, mean age 17.2 years). All participants had normal radiological reports and no participants had a history of brain injury or comorbid neurological disorders. All XXY participants were non-mosaic with a diagnosis by karyotype, and all XY participants were screened to exclude a history of neurodevelopmental or psychiatric disorders. This study was approved by the NIH Combined Neuroscience Institutional Review Board. All participants gave consent or assent, as appropriate. All protocols were completed at the NIH Clinical Center in Bethesda, Maryland.

\section{Cognitive and Behavioral measures}

Full-scale Intelligence Quotient (henceforth “IQ”) was measured for the XXY group using the Wechsler Intelligence Scale for Children, Fifth Edition (WISC-V) and the Wechsler Adult Intelligence Scale, Fourth Edition (WAIS-IV; [31]). Global total psychopathology was measured using age-normed t-scores from the Child Behavior Checklist (CBCL; [32]) and the Adult Behavior Checklist (ABCL; [33]).

\section{Neuroimaging}

Neuroimaging data were collected using a MR750 3-Tesla (General Electric, Wisconsin) whole-body scanner with a 32-channel head coil. High-resolution MP-RAGE T1-weighted anatomical images were collected for each participant (176 contiguous sagittal slices with $256 \mathrm{x}$ 
256 in-plane matrix and $1 \mathrm{~mm}$ slice thickness yielding $1 \mathrm{~mm}$ isotropic voxels). Spontaneous slowly fluctuating brain activity was measured during fMRI through an echo-planar imaging (EPI) sequence while participants were instructed to lay still and focus on a fixation cross for 10 minutes (repetition time of 2 seconds, echo time $=30 \mathrm{~ms}$, flip angle $=60$ degrees, 41 interleaved axial slices per volume, $3 \mathrm{~mm}$ slice thickness in a $216 \mathrm{~mm} \times 216 \mathrm{~mm}$ acquisition matrix, single voxel $=3 \mathrm{~mm}$ isotropic). Resting-state data were evaluated for transient head motion artifacts using the AFNI program@1dDiffMag [34] to quantify the magnitude of head motion throughout the scan $(\mathrm{mm} /$ repetition time $[\mathrm{TR}])$ comparable to average framewise displacement $[35,36]$. Only participants with complete resting-state scans and average head motion $<0.3 \mathrm{~mm} / \mathrm{TR}$ were included.

\section{Preprocessing and analysis of resting-state fMRI data}

Preprocessing was performed using the AFNI software package [34] based on the ANATICOR approach [37]. We removed physiological noise from the echo-planar data by performing principal component analysis (PCA) to identify the first three principal components within ventricles and white matter. We used linear regression to remove these three primary components, head motion, average signal from ventricles, and a local average of white matter signal within a $20 \mathrm{~mm}$ radius centered on each voxel. We then smoothed EPI data with a $6.0 \mathrm{~mm}$ Gaussian kernel and non-linearly transformed scans into standard Talairach space [40] (for more detail see Supplementary Text 1).

We calculated voxel-wise global rsFC maps for each participant by finding the average correlation of the EPI data at one voxel with the time series at every other voxel in the gray matter mask (as described in [30]). For voxels present in 90\% of individual-participant grey matter masks, we Fisher's z-transformed the voxel-wise correlation coefficients and compared groups using two- 
sample t-tests at each voxel while covarying for age and average head motion. We corrected for multiple comparisons across voxels by applying a voxel-level $\mathrm{p}<0.002$ threshold, and a cluster size threshold of 21 -- thereby achieving a corrected FWE of 5\% within the revised version of AFNI's 3dClustSim function, after the bug fix in 2017 [41]. This identified two significant clusters of increased global connectivity in XXY syndrome: a 49-voxel cluster in the left DLPFC and a 22voxel cluster in the right medial temporal lobe.

To ensure the stability of any group difference clusters detected and to eliminate any possibility of noise bias for subsequent seed tests, we implemented a leave-one-out validation technique by recalculating group differences 159 times while iteratively excluding one participant at a time [42]. We identified any clusters that were statistically significant in all 159 iterations as stable clusters of group difference in global connectivity. This procedure -- which screened all gray matter voxels to identify foci with statistically significant and reproducible differences in average rsFC with other brain regions in XXY vs. XY groups - identified a 32-voxel region within the aforementioned left DLPFC cluster showing consistent and significantly increased global connectivity in XXY. By definition, this region was detected in a robust manner that did not depend on noise present in any of the individual datasets. The cluster detected in the medial temporal lobe did not survive leave-one-out validation and was excluded from subsequent analyses.

Next, a seed-based analysis was used to identify the specific patterns of altered interregional connectivity that contributed to the observed 32-voxel cluster of increased DLPFC global connectivity in XXY syndrome. These seed analyses were considered to be statistically independent from the prior screen for altered global rsFC because of the leave-one-out criterion that has been used to define the seed [43]). For each individual, we correlated average EPI time series within the left DLPFC and every other gray matter voxel. Then, after Fisher's z- 
transformation, we performed two-sample t-tests between groups at each voxel, co-varying for participant age and average head motion. This identified the set of target regions which showed altered rsFC with the robust left DLPFC seed in XXY vs. XY groups after correction using the revised version of AFNI's 3dClustsim to ensure FWE of 5\% [41, see above]. Target regions were parcellated using the boundaries along major anatomical divisions and remaining large clusters exceeding the 85 th centile of target cluster size distribution were further divided using a previously-published rsFC parcellation of the brain [44]. This process yielded 39 regions of interest (ROIs), including the original DLPFC seed region. Group differences in rsFC between all unique ROI pairs (i.e. rsFC “edges”) were determined using two-sample t-tests between groups. We considered edges to be statistically significant if they had absolute t-scores above the 95th percentile of an empirical null distribution formed from the most extreme edge-level t-scores from 1000 permutations of group assignment.

\section{Comparing rsFC and anatomical alterations in XXY}

Voxel-wise group differences in gray matter volume (GMV) between XXY and XY groups were estimated using the DARTEL procedure in SPM 12. Images were corrected for magnetic field inhomogeneity, segmented, and normalized to the Montreal Neurological Institute (MNI) space in a unified model. Gray matter probability maps were generated for each individual and smoothed. To find group differences in regional GMV, a voxel-wise ANOVA model in SPM12 was applied to these gray matter probability maps, while covarying for age and total GMV (for more detail, see Supplemental Text 2). The resulting t-maps of XXY vs. XY differences in GMV were corrected for whole-brain comparisons using the revised version of AFNI's 3dClustSim [41] to ensure family-wise error $(\mathrm{FWE})<0.05$. 
We assessed overlap between functional and structural findings by correlating the group difference t-score (XXY vs. XY) of seed-ROI rsFC in each ROI with the group difference t-score (XXY vs. XY) in regional GMV within that ROI. We also tested if the magnitude of inter-regional rsFC disruption in XXY was coupled with the magnitude of inter-regional GMV covariance disruption (see Supplementary Text 3) and found no statistical evidence of such a relationship.

\section{Inter-relating Variation in rsFC and Variation in Clinical Outcomes in XXY}

To assess the role of the left DLPFC connectivity in explaining cognitive and behavioral variation in XXY, we separately conducted Pearson correlations of inter-individual variation in IQ and total CBCL score with inter-individual variation in each gray matter voxel's connectivity with the left DLPFC in XXY. To identify regions showing significant correlations, we applied a clustersize threshold to ensure full FWE $<0.05$ by correcting each individual map to $p<0.025$, accounting for our analysis of both CBCL and IQ ( $p<0.002$, cluster size threshold $=26$ voxels). This thresholding was determined using the revised version of AFNI's 3dClustSim [41].

\section{RESULTS}

\section{Participant characteristics}

Our study cohort included $75 \mathrm{XXY}$ and $84 \mathrm{XY}$ youth aged 6-25 years old (mean age 17 years). Groups did not significantly differ in terms of age $(p=0.45)$ or average head motion $(p=$ 0.12). Mean IQ within the XXY group was $94.9(\mathrm{SD}=12.13)$ and mean total CBCL score was $57.09(\mathrm{SD}=9.20)$. For further details see Supplementary Table 1.

\section{XXY syndrome is associated with a distributed disruption of prefrontal connectivity}

One 32-voxel cluster of increased global rsFC in the left DLPFC in the XXY compared to the XY group survived statistical thresholding and leave-one-out conjunction analyses (see

\section{Methods, Figure 1).}




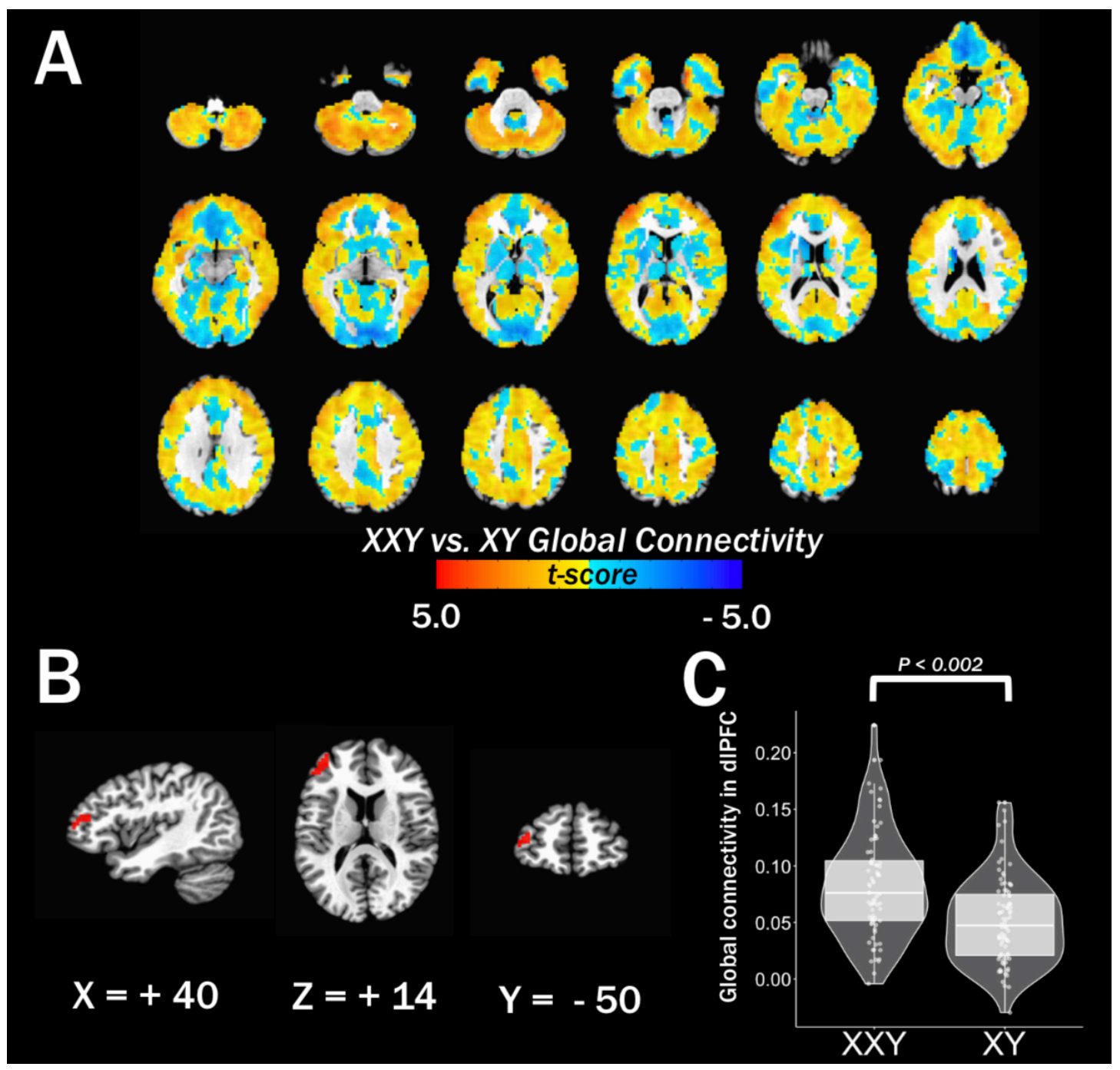

Figure 1. Increased dorsolateral prefrontal cortex global rsFC in XXY syndrome. (A) Unthresholded map of group differences (XXY-XY) in global connectivity while covarying for age and motion effects. (B) Significant 32 voxel cluster of increased global connectivity in the left DLPFC in XXY. Voxels survived all leave-one-out validation permutations at $\mathrm{p}=0.002$, cluster size threshold $=21$ voxels). (C) Average global connectivity in left DLPFC voxels by group.

Seed-based analysis of group differences in voxel-wise connectivity with the left DLPFC revealed 24 clusters showing significantly increased left DLPFC connectivity in XXY syndrome compared to XY controls. Division of these distributed clusters by major anatomical divisions and labelling against an existing brain-wide rsFC parcellation (see Methods) yielded 39 regions of interest (ROIs) for downstream analyses (Figure 2A, Table 1, Supplementary Movie 1). 


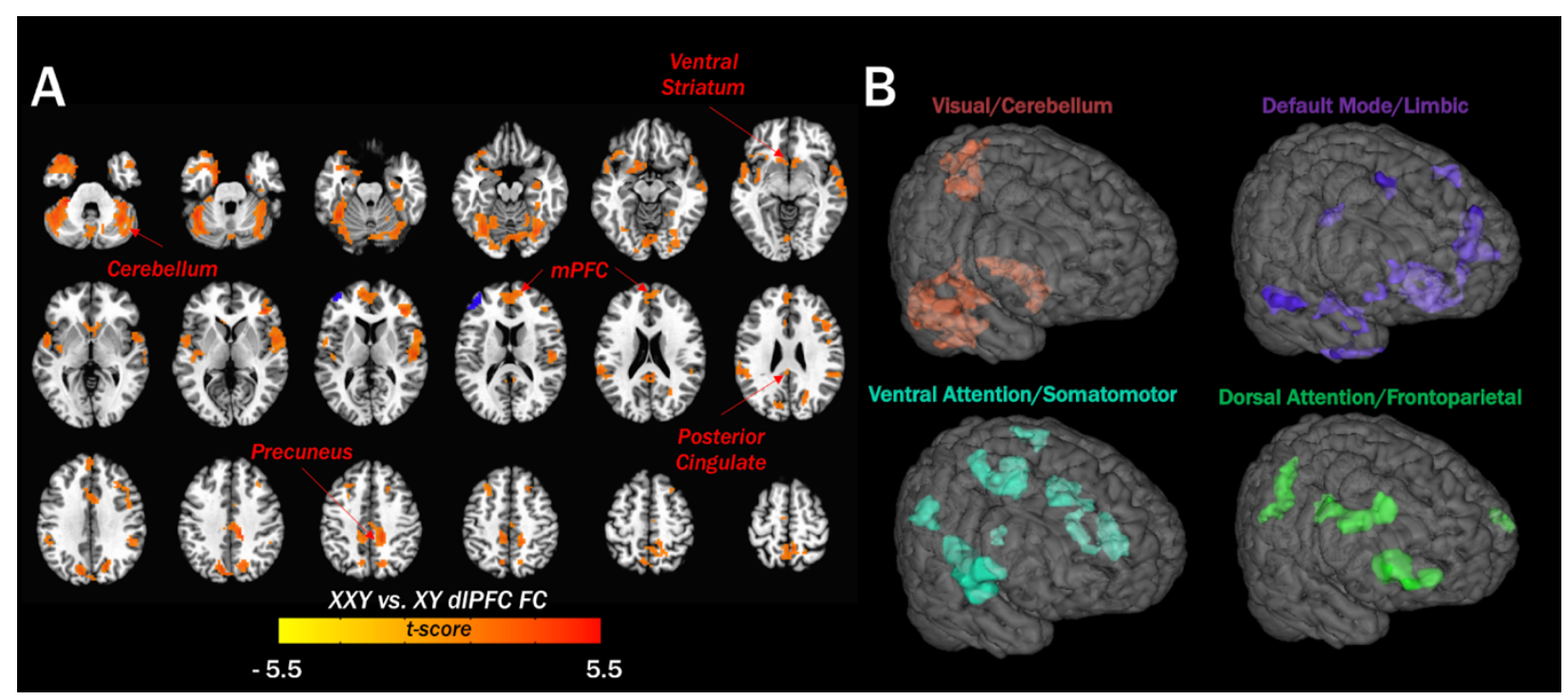

Figure 2. XXY syndrome increased rsFC between the left DLPFC and a distributed set of cortical and subcortical regions. (A) Regions showing significantly increased connectivity (in red) with the left DLPFC seed (in blue) in XXY syndrome relative to XY controls. (B) Volumetric visualization of regions showing increased DLPFC rsFC in XXY syndrome, grouped and colored according to their overlap with canonical resting-state FC networks in the Yeo-Krienen 7-network solution [45]: Red = Visual/Cerebellum, Purple $=$ Default Mode/Limbic, Turquoise $=$ Ventral Attention/Somatomotor, Lime Green $=$ Dorsal Attention/Frontoparietal).

Clustering and annotating these ROIs against classical rsFC networks [45], (see Supplementary Text 4), revealed that altered global rsFC of the left DLPFC in XXY syndrome is driven by dysconnectivity between the left DLPFC and 4 distributed brain systems: [1. Visual/Cerebellum, 2. Default Mode/Limbic, 3. Ventral Attention/Somatomotor, 4. Dorsal Attention/Frontoparietal] (Figure 2B, Supplementary Figure 1). The left DLPFC seed fell within the Frontoparietal network according to this annotation. Permutation testing (see Methods) indicated that dysconnectivity amongst these ROIs was predominantly restricted to edges linked to the left DLPFC cluster of altered global rsFC in XXY syndrome, and some intra-cerebellar and parieto-cerebellar edges (Supplementary Figure 2). 
bioRxiv preprint doi: https://doi.org/10.1101/2020.12.15.422868; this version posted December $16,2020$. The copyright holder for this preprint (which was not certified by peer review) is the author/funder. This article is a US Government work. It is not subject to copyright under 17 USC 105 and is also made available for use under a CCO license.

Resting-state functional connectivity in XXY

Table 1. ROIs showing increased dIPFC connectivity in XXY

\begin{tabular}{|c|c|c|c|c|}
\hline \multirow[t]{2}{*}{ ROI } & \multicolumn{3}{|c|}{ Talairach Coordinates of peak signal } & \multirow[t]{2}{*}{ Voxels $\left(3 \mathrm{~mm}^{3}\right)$} \\
\hline & $\mathbf{X}$ & $\mathbf{Y}$ & $\mathbf{Z}^{\circ}$ & \\
\hline Left Cerebellum (VI) & 37 & 46 & -21 & 233 \\
\hline Right Cerebellum (Crus) & -37 & 52 & -30 & 174 \\
\hline Left Superior Medial Gyrus & 7 & -52 & 11 & 151 \\
\hline Right Cerebellum (VIII) & -28 & 43 & -45 & 150 \\
\hline Left Medial Temporal Pole & 31 & -13 & -33 & 135 \\
\hline Left Cerebellum (X) & 25 & 31 & -33 & 125 \\
\hline Left Superior Temporal Gyrus & 49 & 1 & 2 & 113 \\
\hline Right Heschls Gyrus & -49 & 16 & 11 & 109 \\
\hline Right Cuneus & -16 & 67 & 32 & 108 \\
\hline Right Middle Cingulate Cortex & -13 & 34 & 38 & 99 \\
\hline Right Cerebellum (VI) & -31 & 64 & -18 & 96 \\
\hline Right Inferior Frontal Gyrus & -40 & -34 & 8 & 88 \\
\hline Right Insula Lobe & -49 & -1 & 5 & 86 \\
\hline Left Cuneus & 10 & 73 & 32 & 83 \\
\hline Right Precuneus & -4 & 46 & 56 & 81 \\
\hline Left SupraMarginal Gyrus & 55 & 34 & 23 & 76 \\
\hline Right Inferior Frontal Gyrus & -43 & -13 & 26 & 76 \\
\hline Right Olfactory Cortex/Caudate & -7 & -10 & -6 & 76 \\
\hline Right Middle Temporal Gyrus & -61 & 13 & -6 & 63 \\
\hline Right Inferior Temporal Gyrus & -37 & -1 & -36 & 61 \\
\hline Right ParaHippocampal Gyrus & -19 & 4 & -24 & 61 \\
\hline Left ParaHippocampal Gyrus & 16 & -1 & -24 & 58 \\
\hline Left Heschls Gyrus & 40 & 19 & 8 & 55 \\
\hline Left Posterior Cingulate Cortex & 4 & 37 & 23 & 52 \\
\hline Left Cerebellum (VIII) & 4 & 61 & -33 & 44 \\
\hline Right SupraMarginal Gyrus & -46 & 37 & 29 & 43 \\
\hline Left Middle Cingulate Cortex & 4 & 37 & 44 & 42 \\
\hline Left Inferior Frontal Gyrus & 31 & -25 & -15 & 42 \\
\hline Left Supplementary Motor Area & 7 & 13 & 62 & 38 \\
\hline Left Middle Frontal Gyrus & 22 & -25 & 41 & 37 \\
\hline Right Anterior Cingulate Cortex & -4 & -7 & 29 & 35 \\
\hline Left Middle Frontal Gyrus & 40 & -49 & 11 & 32 \\
\hline Right Middle Frontal Gyrus & -22 & -19 & 44 & 29 \\
\hline Left Cerebellum (VI) & 19 & 58 & -15 & 29 \\
\hline Left Middle Occipital Gyrus & 22 & 79 & 8 & 23 \\
\hline Right Superior Occipital Gyrus & -25 & 70 & 17 & 23 \\
\hline Right Cerebellum (VIII) & -31 & 49 & -45 & 23 \\
\hline Right Insula Lobe/Olfactory/Putamen & -28 & -10 & -6 & 22 \\
\hline Left Lingual Gyrus & 10 & 55 & 0 & 21 \\
\hline
\end{tabular}

Note: ROI names determined by anatomical location of the voxel of greatest group difference in dlPFC connectivity according to the Eickhoff-Zilles macro labels in Talairach space [46]. 
bioRxiv preprint doi: https://doi.org/10.1101/2020.12.15.422868; this version posted December 16, 2020. The copyright holder for this preprint (which was not certified by peer review) is the author/funder. This article is a US Government work. It is not subject to copyright under 17 USC 105 and is also made available for use under a CCO license.

Resting-state functional connectivity in XXY

\section{Changes in rsFC and regional anatomy are partially coupled in XXY syndrome}

Voxel-wise analysis of group differences in GMV (controlling for age and total GMV) revealed relative volumetric expansion in parieto-occipital regions alongside relative volumetric reduction in cerebellum, subcortex, prefrontal cortex, and medial temporal lobes $(p<0.001$, cluster size threshold $=3061 \mathrm{~mm}$ isotropic voxels; Figure 3A, Supplementary Table 2). To test the interrelationship between altered rsFC and altered GMV in XXY syndrome, we asked if inter-ROI variation in the magnitude of altered DLPFC connectivity was correlated with inter-ROI variation in the (unsigned) magnitude of GMV group differences (Methods). The magnitude of group differences in ROI rsFC dysconnectivity with the left DLPFC in XXY syndrome was positively associated with the magnitude of group differences in VBM using robust correlation $(r=0.47$; Figure 3B). This correlation was statistically significant compared to a null distribution of correlations from group permutation $\left(p_{\text {perm }}=0.02\right.$; see Supplementary Text 5). Thus, brain regions showing greater dysconnectivity with the DLPFC in XXY syndrome also tend to be those that show greater group-level alterations of relative GMV.

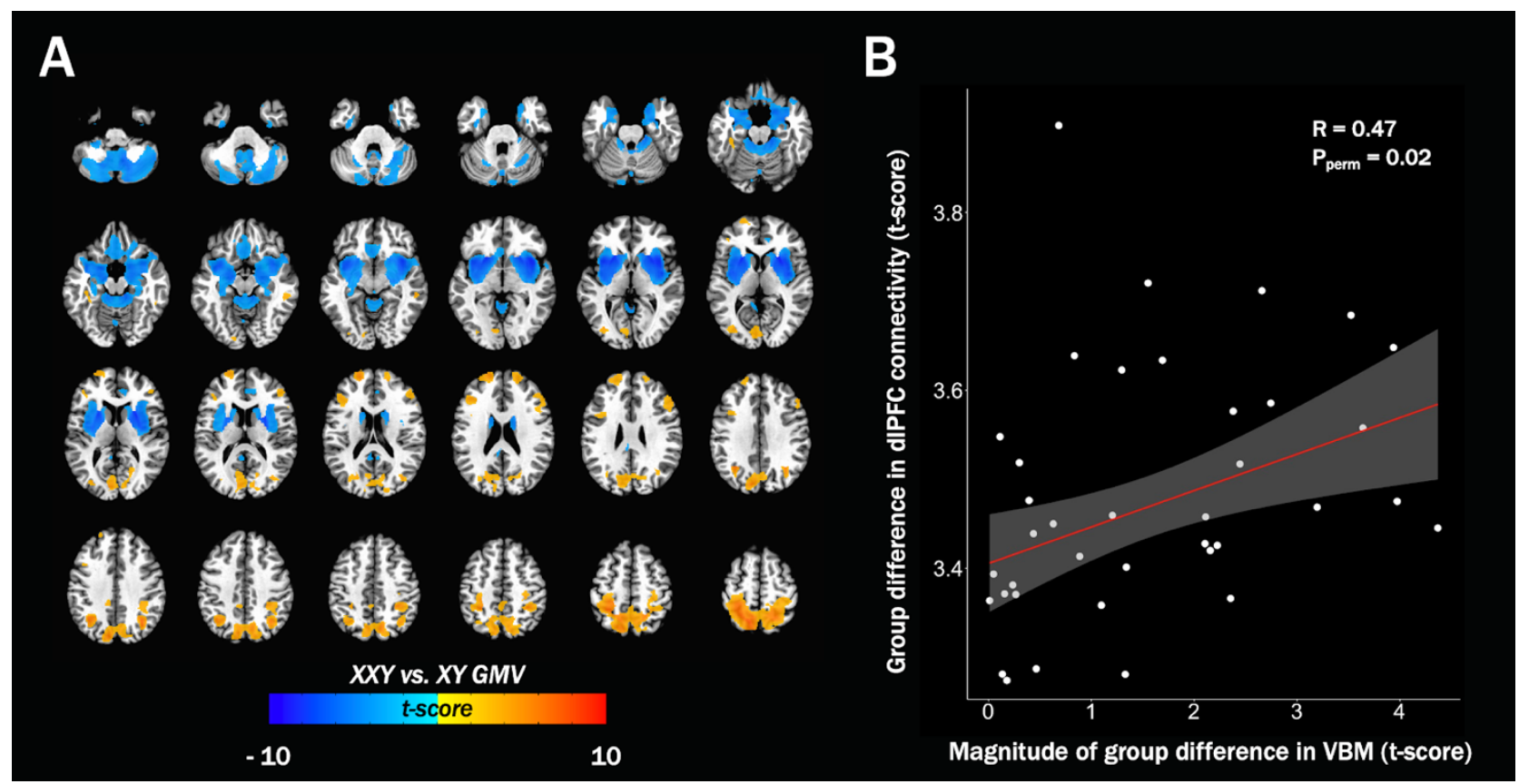


Figure 3. Altered regional brain volume in XXY syndrome and its relationship with rsFC changes. (A) Volumetric group differences in $(X X Y-X Y ; p<0.002$, cluster size threshold $=3061 \mathrm{~mm}$ isotropic voxels). (B) Scatterplot showing correlation between group difference in DLPFC connectivity (XXY-XY) and magnitude of group difference in VBM (XXY-XY) at each functionally defined ROI.

\section{Prefrontal connectivity is negatively correlated with psychopathology in XXY}

Whole-brain voxel-wise correlations between inter-individual variation of left DLPFC rsFC and inter-individual variation in total CBCL score identified clusters of negative correlation in the left precuneus and in the bilateral inferior temporal gyri (ITG) $(p<.002, \mathrm{FWE}=0.025$; Peak $r$ values: precuneus $r=-0.45$, Left ITG $r=-0.48$, Right ITG $r=-0.43$; Figure 4A). Conjunction with the map of altered DLPFC connectivity in XXY syndrome (Figure 2A) identified an overlap in the right precuneus -- indicating that this region not only shows increased connectivity with the DLPFC in XXY syndrome as compared to XY controls, but that also amongst individuals with XXY syndrome, greater DLPFC-precuneus connectivity is associated with less severe psychopathology. No clusters showed significant correlation between DLPFC connectivity and IQ. To better qualify our findings for rsFC-CBCL relationships in XYY syndrome, we also conducted the same analysis in the pediatric subset of XY controls with available CBCL data, and found that clusters identified in XXY syndrome were nonsignificantly positively correlated with total CBCL scores in euploidic male controls (see Supplementary Text 6). Secondly, to gauge relative contributions of each CBCL subscale, we conducted sparse canonical correlation analysis (sCCA) between significant voxels in the XXY group and subscales of the CBCL. We did not observe that the correlation with total psychopathology scores was driven by specific subscales of the CBCL (see Supplementary Text 6). 


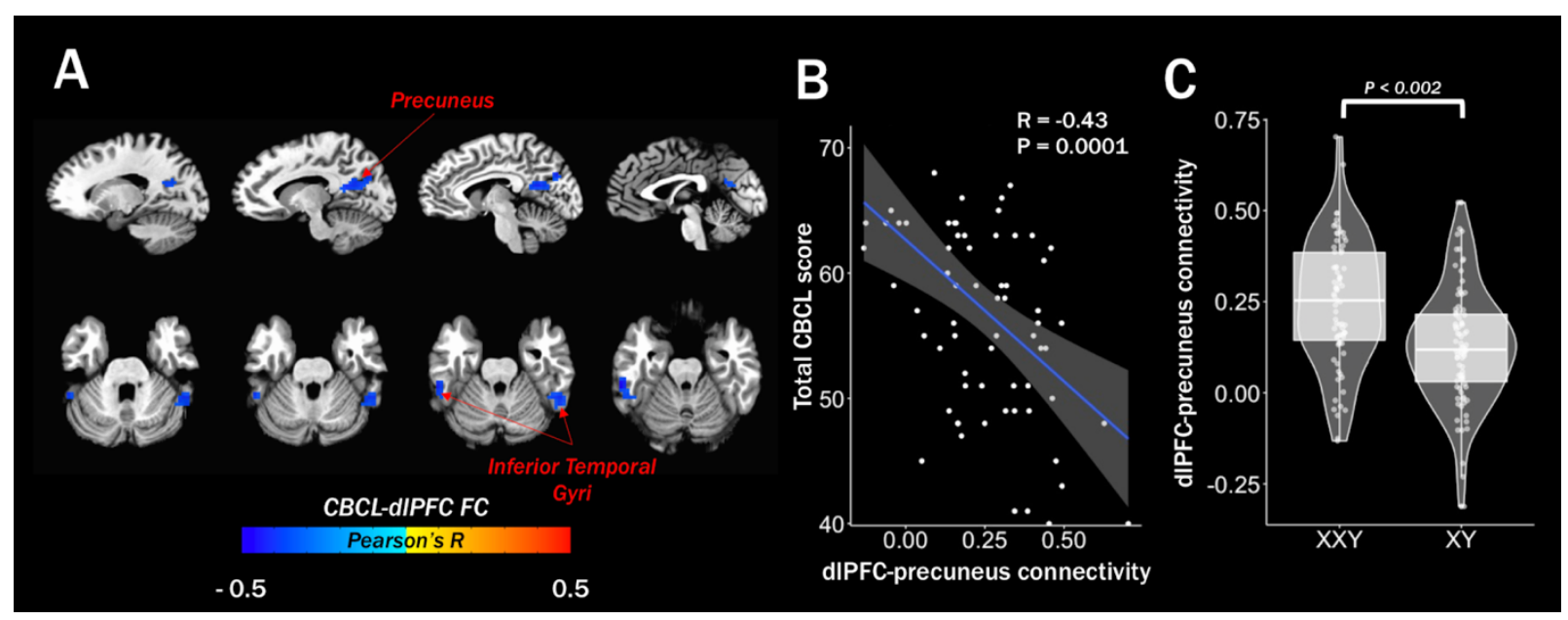

Figure 4. Relationships between DLPFC FC and psychopathology in XXY syndrome. (A) Regions showing significant correlations between DLPFC connectivity and total CBCL score in XXY syndrome $(\mathrm{p}<0.002$, cluster size threshold $=26$ voxels, FWE $=0.025)$. (B) Scatterplot showing inverse correlation between DLPFC connectivity and total CBCL score among precuneus voxels that also show significant DLPFC increase in XXY. (C). Boxplot showing average DLPFC connectivity in these precuneus voxels by group.

\section{DISCUSSION}

Our study provides the first whole-brain screen for rsFC alterations in XXY, and their links with brain structure and behavioral measurements. We provide evidence for widespread disruption in functional brain organization in XXY that is partly coupled with anatomical alterations and related to the severity of overall psychopathology. These results inform our understanding of XXY syndrome as a medical condition in its own right, and as a genetics-first model for mechanisms of genetic risk in psychiatry more generally.

\section{Prefrontal dysconnectivity in XXY syndrome}

We found significantly increased global connectivity in the left DLPFC in XXY which was associated with overconnectivity with a distributed set of cortical, subcortical, and cerebellar regions. These regions collectively encompass several major canonical rsFC networks within the human brain [45]. This pattern of altered rsFC in XXY syndrome connects with existing literature in several notable ways. 
First, many of the regions showing overconnectivity with the DLPFC in XXY syndrome have been reported to show prefrontal underconnectivity in Turner syndrome $(45, \mathrm{X})$ [DLPFCintraparietal sulcus [11], DLPFC-angular gyrus [47]]. These inverted disruptions of DLPFC rsFC in XXY and Turner syndrome strengthen the case for a direct effect of X-chromosome dosage on frontoparietal rsFC organization of the human brain. Second, the nature of rsFC alterations observed in XXY syndrome within the current study shows a mixed pattern of partial overlap and divergence with those seen in other genetically defined neuropsychiatric disorders (specific comparisons provided in Supplementary Text 7). Consequently, an important goal for future work will be explicitly testing this hypothesis in prospectively designed comparative studies of rsFC across multiple neurogenetic disorders. Third, abnormalities of DLPFC rsFC, such as those we report here in XXY syndrome, have also been associated with diverse idiopathic neuropsychiatric disorders [48-51]. This observation builds on the occurrence of DLPFC rsFC alterations across diverse genetically defined neuropsychiatric disorders (see above) and may reflect the role of DLPFC in those higher-order domains of brain function [52] which are core to the manifestation of diverse psychiatric presentations. Given the position of the DLPFC towards the peak of the functional hierarchy [53], disruption may alter connectivity amongst the functionally disparate regions we observed. Finally, longitudinal studies of rsFC in typically developing youth have revealed substantial reorganization of DLPFC connectivity with other brain regions en route to adulthood $[54,55]$. In this context, our findings in XXY syndrome could potentially be conceptualized as a shift in the timing of a normative developmental progression in rsFC, although a formal test of this hypothesis must await the accumulation of later longitudinal rs-fMRI datasets in XXY syndrome. 


\section{Partial convergence of rsFC and anatomical changes in XXY syndrome}

For regions showing altered rsFC with the DLPFC in XXY syndrome, we observed that interregional variation in the magnitude of rsFC dysconnectivity was correlated with the absolute magnitude of regional brain volume change -- indicating that disruptions of rsFC in $\mathrm{XXY}$ syndrome may be mechanistically linked to disruptions of anatomy that are visible in vivo. Our study design (and observational methods in humans generally) prevents direct analysis of causality, although longitudinal data could potentially support a causal relationship. It is also possible that XXY syndrome induces a coordinated disruption of both these brain phenotypes without one necessarily driving the other. Interestingly, in vivo imaging has also detected overlapping alterations of brain structure and function in other genetically defined [e.g. 22q11 copy number variations, [19]] and idiopathic [e.g. ASD [56] and attention-deficit/hyperactivity disorder [57]] neuropsychiatric disorders. Thus, although such overlaps are certainly not a general rule across psychiatric disorders $[58,59]$-- our findings motivate broader multimodal analyses of regional brain disruption on neuropsychiatric disorders.

\section{Severity of psychopathology in XXY correlates with variation in DLPFC rsFC}

We observed that variation in total psychopathology, but not in IQ, correlates with DLPFC rsFC in XXY syndrome. Strikingly, comparison with prior work suggests that prefrontal dysconnectivity is a correlate of psychopathology among multiple neurogenetic conditions, idiopathic psychiatric disorders, and in healthy populations $[14,16,20,21,60,61]$. We find DLPFC rsFC correlates with total psychopathology in XXY syndrome within the inferior temporal gyrus (ITG) and the precuneus. The functioning of the ITG has been associated with higher order social communication [62] and visual processing [63]. The precuneus is a key node within the brain's default mode network and implicated in several higher order capacities including working 
memory and self-directed cognition [64]. The precuneus focus where DLPFC rsFC connectivity correlates negatively with psychopathology in XXY is notable for also showing increased DLPFC rsFC in XXY syndrome as compared to XY controls. Thus, the "direction" of group differences and within-group symptom correlation for DLPFC-precuneus rsFC in XXY syndrome appear to be opposing. This preliminary finding awaits replication in independent cohorts but could potentially represent a compensatory process such as has been reported in other disorders $[65,66]$. Importantly, the pattern of CBCL correlation with this edge in XYY syndrome was both qualitatively and quantitatively distinct from that in the XY group and contributed to by multiple CBCL subscales. An important caveat is that CBCL data was only available on XY participants under $18(\mathrm{~N}=45)$, therefore we cannot rule out confounding age differences in this analysis.

\section{Limitations and future directions}

Our findings should be considered with a set of important caveats and limitations in addition to those already mentioned. Firstly, as our sample is not population based, we cannot assume that the phenotype of XXY in this sample is fully representative of all cases of XXY. Indeed, as a large number of individuals with XXY go undiagnosed, it is possible that those with higher penetrance are more frequently diagnosed, and therefore a more extreme phenotype is overrepresented in our sample. This is a known problem in the SCA literature and clinical studies of genetically defined neuropsychiatric disorders more broadly $[5,67,68]$. Second, carriage of a supernumerary $\mathrm{X}$-chromosome in males is also associated with hypogonadism and testosterone deficiency beginning at the onset of puberty [69]. Future research should assess the degree to which hormonal influence could act as a mediating variable for SCA on brain function. Third, recent studies have argued that substantially larger sample sizes are necessary to reliably correlate brain function with behavior $[60,70]$. Collecting data from such a large sample is infeasible when 
studying rare neurogenetic conditions such as XXY. Therefore, future research should seek to independently reproduce brain-behavior correlations observed here to ensure their reliability.

\section{Summary}

Notwithstanding these limitations, our study provides the first systematic investigation of brain connectivity in XXY syndrome -- which represents a common and clinically significant genetic risk for neuropsychiatric impairments. In doing so, we identify a distributed pattern of altered prefrontal rsFC in $\mathrm{XXY}$ syndrome compared to $\mathrm{XY}$ males. These changes in brain connectivity are partly correlated with regional anatomical disruptions in XXY syndrome, and also overlap with regions where variation in prefrontal connectivity is correlated with variation in psychopathology amongst affected individuals. Taken together, these findings provide a fuller understanding of functional and structural brain changes in XXY syndrome as a medical disorder in its own right, and as a "genetics-first" model for neuropsychiatric impairment more broadly.

\section{ACKNOWLEDGEMENTS}

The authors would like to thank the patients and their families for their participation in this study and the Association for X and Y Chromosome Variations (AXYS; https://genetic.org) for their assistance in recruitment efforts. All research was funded by the NIMH Intramural Research Program. (Clinical trial reg. No. NCT00001246; clinicaltrials.gov; NIH Annual Report Number, ZIAMH002949-03; Protocol number: 89-M-0006).

\section{CONFLICT OF INTEREST}

The authors declare no conflicts of interest. 


\section{REFERENCES}

1. Coffee B, Keith K, Albizua I, Malone T, Mowrey J, Sherman SL, et al. Incidence of fragile X syndrome by newborn screening for methylated FMR1 DNA. Am J Hum Genet. 2009;85:503514.

2. Leggett V, Jacobs P, Nation K, Scerif G, Bishop DVM. Neurocognitive outcomes of individuals with a sex chromosome trisomy: XXX, XYY, or XXY: a systematic review*: Systematic Review. Developmental Medicine \& Child Neurology. 02/2010;52:119-129.

3. Tartaglia N, Cordeiro L, Howell S, Wilson R, Janusz J. The spectrum of the behavioral phenotype in boys and adolescents 47,XXY (Klinefelter syndrome). Pediatr Endocrinol Rev. 2010;8 Suppl 1:151-159.

4. Ross JL, Roeltgen DP, Kushner H, Zinn AR, Reiss A, Bardsley MZ, et al. Behavioral and Social Phenotypes in Boys With 47,XYY Syndrome or 47,XXY Klinefelter Syndrome. Pediatrics. 04/2012;129:769-778.

5. van Rijn S. A review of neurocognitive functioning and risk for psychopathology in sex chromosome trisomy (47,XXY, 47,XXX, 47, XYY). Curr Opin Psychiatry. 2019;32:79-84.

6. Raznahan A, Lee NR, Greenstein D, Wallace GL, Blumenthal JD, Clasen LS, et al. Globally Divergent but Locally Convergent X- and Y-Chromosome Influences on Cortical Development. Cereb Cortex. 2016;26:70-79.

7. Bryant DM, Hoeft F, Lai S, Lackey J, Roeltgen D, Ross J, et al. Neuroanatomical phenotype of Klinefelter syndrome in childhood: a voxel-based morphometry study. J Neurosci. 
8. Mankiw C, Park MTM, Reardon PK, Fish AM, Clasen LS, Greenstein D, et al. Allometric Analysis Detects Brain Size-Independent Effects of Sex and Sex Chromosome Complement on Human Cerebellar Organization. J Neurosci. 2017;37:5221-5231.

9. Marzelli MJ, Hoeft F, Hong DS, Reiss AL. Neuroanatomical spatial patterns in Turner syndrome. Neuroimage. 2011;55:439-447.

10. Hallahan BP, Craig MC, Toal F, Daly EM, Moore CJ, Ambikapathy A, et al. In vivo brain anatomy of adult males with Fragile X syndrome: an MRI study. Neuroimage. 2011;54:16-24.

11. Green T, Saggar M, Ishak A, Hong DS, Reiss AL. X-Chromosome Effects on Attention Networks: Insights from Imaging Resting-State Networks in Turner Syndrome. Cereb Cortex. $2018 ; 28: 3176-3183$.

12. Debbané M, Lazouret M, Lagioia A, Schneider M, Van De Ville D, Eliez S. Resting-state networks in adolescents with 22q11.2 deletion syndrome: associations with prodromal symptoms and executive functions. Schizophr Res. 2012;139:33-39.

13. Moreau C, Urchs S, Orban P, Schramm C, Dumas G, Labbe A, et al. Neuropsychiatric mutations delineate functional brain connectivity dimensions contributing to autism and schizophrenia. 2019:862615.

14. Schreiner MJ, Karlsgodt KH, Uddin LQ, Chow C, Congdon E, Jalbrzikowski M, et al. Default mode network connectivity and reciprocal social behavior in 22q11.2 deletion syndrome. Soc Cogn Affect Neurosci. 2014;9:1261-1267.

15. Zöller D, Schaer M, Scariati E, Padula MC, Eliez S, Van De Ville D. Disentangling resting-state BOLD variability and PCC functional connectivity in 22q11.2 deletion syndrome. Neuroimage. 2017;149:85-97. 
16. Bertero A, Liska A, Pagani M, Parolisi R, Masferrer ME, Gritti M, et al. Autism-associated 16p11.2 microdeletion impairs prefrontal functional connectivity in mouse and human. Brain. 2018;141:2055-2065.

17. Hall SS, Jiang H, Reiss AL, Greicius MD. Identifying large-scale brain networks in fragile X syndrome. JAMA Psychiatry. 2013;70:1215-1223.

18. Raznahan A. Genetics-First Approaches in Biological Psychiatry. Biol Psychiatry. 2018;84:234235.

19. Padula MC, Schaer M, Scariati E, Schneider M, Van De Ville D, Debbané M, et al. Structural and functional connectivity in the default mode network in $22 \mathrm{q} 11.2$ deletion syndrome. $\mathrm{J}$ Neurodev Disord. 2015;7:23.

20. Mattiaccio LM, Coman IL, Schreiner MJ, Antshel KM, Fremont WP, Bearden CE, et al. Atypical functional connectivity in resting-state networks of individuals with $22 \mathrm{q} 11.2$ deletion syndrome: associations with neurocognitive and psychiatric functioning. J Neurodev Disord. 2016;8:2.

21. Zöller D, Sandini C, Karahanoğlu FI, Padula MC, Schaer M, Eliez S, et al. Large-Scale Brain Network Dynamics Provide a Measure of Psychosis and Anxiety in 22q11.2 Deletion Syndrome. Biol Psychiatry Cogn Neurosci Neuroimaging. 2019;4:881-892.

22. Arnett AB, Pennington BF, Willcutt EG, DeFries JC, Olson RK. Sex differences in ADHD symptom severity. J Child Psychol Psychiatry. 2015;56:632-639.

23. Mandy W, Chilvers R, Chowdhury U, Salter G, Seigal A, Skuse D. Sex Differences in Autism Spectrum Disorder: Evidence from a Large Sample of Children and Adolescents. J Autism Dev Disord. 7/2012;42:1304-1313. 
24. Angst J, Gamma A, Gastpar M, Lépine J-P, Mendlewicz J, Tylee A, et al. Gender differences in depression. Epidemiological findings from the European DEPRES I and II studies. Eur Arch Psychiatry Clin Neurosci. 2002;252:201-209.

25. McLean CP, Asnaani A, Litz BT, Hofmann SG. Gender differences in anxiety disorders: prevalence, course of illness, comorbidity and burden of illness. J Psychiatr Res. 2011;45:10271035.

26. Lotze M, Domin M, Gerlach FH, Gaser C, Lueders E, Schmidt CO, et al. Novel findings from 2,838 Adult Brains on Sex Differences in Gray Matter Brain Volume. Sci Rep. 12/2019;9:1671.

27. Liu S, Seidlitz J, Blumenthal JD, Clasen LS, Raznahan A. Integrative structural, functional, and transcriptomic analyses of sex-biased brain organization in humans. Proc Natl Acad Sci U S A. 2020;117:18788-18798.

28. Nguyen DK, Disteche CM. High expression of the mammalian X chromosome in brain. Brain Res. 12/2006;1126:46-49.

29. Neri G, Schwartz CE, Lubs HA, Stevenson RE. X-linked intellectual disability update 2017. Am J Med Genet A. 06 2018;176:1375-1388.

30. Gotts SJ, Simmons WK, Milbury LA, Wallace GL, Cox RW, Martin A. Fractionation of social brain circuits in autism spectrum disorders. Brain. 9/2012;135:2711-2725.

31. Jacobson LA, Mahone EM. Wechsler Intelligence Scale for Children. In: Kreutzer JS, DeLuca J, Caplan B, editors. Encyclopedia of Clinical Neuropsychology, New York, NY: Springer New York; 2011. p. 2682-2688.

32. Achenbach TM. Child Behavior Checklist. In: Kreutzer JS, DeLuca J, Caplan B, editors. Encyclopedia of Clinical Neuropsychology, New York, NY: Springer New York; 2011. p. 546552. 
33. Rescorla LA, Achenbach TM. The Achenbach System of Empirically Based Assessment (ASEBA) for Ages 18 to 90 Years. The Use of Psychological Testing for Treatment Planning and Outcomes Assessment: Instruments for Adults, Volume 3, 3rd Ed. 1008;3:115-152.

34. Cox RW. AFNI: software for analysis and visualization of functional magnetic resonance neuroimages. Comput Biomed Res. 1996;29:162-173.

35. Power JD, Barnes KA, Snyder AZ, Schlaggar BL, Petersen SE. Spurious but systematic correlations in functional connectivity MRI networks arise from subject motion. Neuroimage. $2012 ; 59: 2142-2154$.

36. Berman RA, Gotts SJ, McAdams HM, Greenstein D, Lalonde F, Clasen L, et al. Disrupted sensorimotor and social-cognitive networks underlie symptoms in childhood-onset schizophrenia. Brain. 2016;139:276-291.

37. Jo HJ, Saad ZS, Simmons WK, Milbury LA, Cox RW. Mapping sources of correlation in resting state FMRI, with artifact detection and removal. Neuroimage. 2010;52:571-582.

38. Stoddard J, Gotts SJ, Brotman MA, Lever S, Hsu D, Zarate C, et al. Aberrant intrinsic functional connectivity within and between corticostriatal and temporal-parietal networks in adults and youth with bipolar disorder. Psychol Med. 2016;46:1509-1522.

39. Behzadi Y, Restom K, Liau J, Liu TT. A component based noise correction method (CompCor) for BOLD and perfusion based fMRI. Neuroimage. 2007;37:90-101.

40. Talairach J, Tournoux P. Co-planar Stereotaxic Atlas of the Human Brain: 3-dimensional Proportional System : an Approach to Cerebral Imaging. G. Thieme; 1988.

41. Cox RW, Chen G, Glen DR, Reynolds RC, Taylor PA. fMRI clustering and false-positive rates. Proc Natl Acad Sci U S A. 2017;114:E3370-E3371. 
42. Smith REW, Avery JA, Wallace GL, Kenworthy L, Gotts SJ, Martin A. Sex Differences in Resting-State Functional Connectivity of the Cerebellum in Autism Spectrum Disorder. Front Hum Neurosci. 2019;13:104.

43. Vul E, Harris C, Winkielman P, Pashler H. Puzzlingly High Correlations in fMRI Studies of Emotion, Personality, and Social Cognition. Perspect Psychol Sci. 2009;4:274-290.

44. Bellec P, Rosa-Neto P, Lyttelton OC, Benali H, Evans AC. Multi-level bootstrap analysis of stable clusters in resting-state fMRI. Neuroimage. 2010;51:1126-1139.

45. Yeo BT, Krienen FM, Sepulcre J, Sabuncu MR, Lashkari D, Hollinshead M, et al. The organization of the human cerebral cortex estimated by intrinsic functional connectivity. J Neurophysiol. 09/2011;106:1125-1165.

46. Eickhoff SB, Stephan KE, Mohlberg H, Grefkes C, Fink GR, Amunts K, et al. A new SPM toolbox for combining probabilistic cytoarchitectonic maps and functional imaging data. Neuroimage. 2005;25:1325-1335.

47. Xie S, Yang J, Zhang Z, Zhao C, Bi Y, Zhao Q, et al. The Effects of the X Chromosome on Intrinsic Functional Connectivity in the Human Brain: Evidence from Turner Syndrome Patients. Cereb Cortex. 2015;27:474-484.

48. Cubillo A, Halari R, Smith A, Taylor E, Rubia K. A review of fronto-striatal and fronto-cortical brain abnormalities in children and adults with Attention Deficit Hyperactivity Disorder (ADHD) and new evidence for dysfunction in adults with ADHD during motivation and attention. Cortex. 2012;48:194-215.

49. Meyer-Lindenberg AS, Olsen RK, Kohn PD, Brown T, Egan MF, Weinberger DR, et al. Regionally specific disturbance of dorsolateral prefrontal-hippocampal functional connectivity in schizophrenia. Arch Gen Psychiatry. 2005;62:379-386. 
50. Avissar M, Powell F, Ilieva I, Respino M, Gunning FM, Liston C, et al. Functional connectivity of the left DLPFC to striatum predicts treatment response of depression to TMS. Brain Stimul. 2017;10:919-925.

51. Ibrahim K, Eilbott JA, Ventola P, He G, Pelphrey KA, McCarthy G, et al. Reduced AmygdalaPrefrontal Functional Connectivity in Children With Autism Spectrum Disorder and Cooccurring Disruptive Behavior. Biol Psychiatry Cogn Neurosci Neuroimaging. 2019;4:10311041.

52. Niendam TA, Laird AR, Ray KL, Dean YM, Glahn DC, Carter CS. Meta-analytic evidence for a superordinate cognitive control network subserving diverse executive functions. Cogn Affect Behav Neurosci. 2012;12:241-268.

53. Margulies DS, Ghosh SS, Goulas A, Falkiewicz M, Huntenburg JM, Langs G, et al. Situating the default-mode network along a principal gradient of macroscale cortical organization. Proc Natl Acad Sci U S A. 2016;113:12574-12579.

54. David S. Grayson DAF. Development of large-scale functional networks from birth to adulthood: a guide to neuroimaging literature. Neuroimage. 2017;160:15.

55. Gu S, Satterthwaite TD, Medaglia JD, Yang M, Gur RE, Gur RC, et al. Emergence of system roles in normative neurodevelopment. Proc Natl Acad Sci U S A. 2015;112:13681-13686.

56. Wang J, Fu K, Chen L, Duan X, Guo X, Chen H, et al. Increased Gray Matter Volume and Resting-State Functional Connectivity in Somatosensory Cortex and their Relationship with Autistic Symptoms in Young Boys with Autism Spectrum Disorder. Front Physiol. 2017;8:588.

57. Wu Z-M, Llera A, Hoogman M, Cao Q-J, Zwiers MP, Bralten J, et al. Linked anatomical and functional brain alterations in children with attention-deficit/hyperactivity disorder. Neuroimage Clin. 2019;23:101851. 
58. Zhuo C, Zhu J, Wang C, Qu H, Ma X, Qin W. Different spatial patterns of brain atrophy and global functional connectivity impairments in major depressive disorder. Brain Imaging Behav. 2017;11:1678-1689.

59. Wang L, Zhao Y, Edmiston EK, Womer FY, Zhang R, Zhao P, et al. Structural and Functional Abnormities of Amygdala and Prefrontal Cortex in Major Depressive Disorder With Suicide Attempts. Front Psychiatry. 2019;10:923.

60. Marek S, Tervo-Clemmens B, Calabro FJ, Montez DF, Kay BP, Hatoum AS, et al. Towards Reproducible Brain-Wide Association Studies. Neuroscience; 2020.

61. Xia CH, Ma Z, Ciric R, Gu S, Betzel RF, Kaczkurkin AN, et al. Linked dimensions of psychopathology and connectivity in functional brain networks. Nat Commun. 2018;9:3003.

62. Xu J, Gannon PJ, Emmorey K, Smith JF, Braun AR. Symbolic gestures and spoken language are processed by a common neural system. Proc Natl Acad Sci U S A. 2009;106:20664-20669.

63. Ishai A, Ungerleider LG, Martin A, Schouten JL, Haxby JV. Distributed representation of objects in the human ventral visual pathway. Proc Natl Acad Sci U S A. 1999;96:9379-9384.

64. Margulies DS, Vincent JL, Kelly C, Lohmann G, Uddin LQ, Biswal BB, et al. Precuneus shares intrinsic functional architecture in humans and monkeys. Proc Natl Acad Sci U S A. 2009;106:20069-20074.

65. Saggar M, Tsalikian E, Mauras N, Mazaika P, White NH, Weinzimer S, et al. Compensatory Hyperconnectivity in Developing Brains of Young Children With Type 1 Diabetes. Diabetes. 2017;66:754-762.

66. Han SD, Bangen KJ, Bondi MW. Functional magnetic resonance imaging of compensatory neural recruitment in aging and risk for Alzheimer's disease: review and recommendations. Dement Geriatr Cogn Disord. 2009;27:1-10. 
67. Bardsley MZ, Kowal K, Levy C, Gosek A, Ayari N, Tartaglia N, et al. 47,XYY syndrome: clinical phenotype and timing of ascertainment. J Pediatr. 2013;163:1085-1094.

68. Gunther DF, Eugster E, Zagar AJ, Bryant CG, Davenport ML, Quigley CA. Ascertainment bias in Turner syndrome: new insights from girls who were diagnosed incidentally in prenatal life. Pediatrics. 2004;114:640-644.

69. Bonomi M, Rochira V, Pasquali D, Balercia G, Jannini EA, Ferlin A, et al. Klinefelter syndrome (KS): genetics, clinical phenotype and hypogonadism. J Endocrinol Invest. 2017;40:123-134.

70. Helmer M, Warrington S, Mohammadi-Nejad A-R, Ji JL, Howell A, Rosand B, et al. On stability of Canonical Correlation Analysis and Partial Least Squares with application to brain-behavior associations. 2020:2020.08.25.265546. 